\title{
Enhanced Disease Surveillance during the 2012 Republican National Convention, Tampa, FL
}

\author{
David Atrubin*, Michael Wiese, Rebecca Snider, Kiley Workman and Warren McDougle \\ Hillsborough County Health Department, Tampa, FL, USA
}

\section{Objective}

To describe disease and illness surveillance utilized during the 2012 Republican National Convention (RNC) held August 26-30, 2012 in Tampa, FL.

\section{Introduction}

While the Tampa Bay Area has previously hosted other high profile events that required heightened disease surveillance (e.g., two Super Bowls), the 2012 RNC marked the first national special security event (NSSE) held in Florida. The Hillsborough County Health Department (HCHD), in conjunction with the Pinellas County Health Department (PinCHD) coordinated disease surveillance activities during this time frame. This presentation will focus of the disease surveillance efforts of the Hillsborough County Health Department during the 2012 RNC.

In addition to the surveillance systems that are used routinely, the HCHD Epidemiology Program implemented additional systems designed to rapidly detect individual cases and outbreaks of public health importance. The short duration of RNC, coupled with the large number of visitors to our area, provided additional surveillance challenges.

Tropical Storm Isaac, which threatened Tampa in the days leading up to RNC, and an overwhelming law enforcement presence likely dissuaded many protestors from coming to Tampa. As a result, a tiny fraction of the number of protestors that were expected actually showed up.

\section{Methods}

Our normal daily analysis of the emergency department (ED) data using the Electronic Surveillance System for the Early Notification of Community-based Epidemics (ESSENCE) was expanded to look in detail at ED volumes and chief complaints of those patients who live outside of a 5-county Tampa Bay area. This analysis used patient zip code to determine place of residence. Additionally, ESSENCE queries were utilized to look for heat, tear gas, and RNC related exposures. The ESSENCE system also receives Poison Control data every 15 minutes. Expanded analyses of the Poison Control data were conducted as well. Two Disaster Medical Assistance Teams (DMATs) were deployed in Tampa during the RNC. Data was collected electronically and transmitted through ESSENCE as well.

The HCHD also asked infection preventionists, health care providers, hotels, labs, and Mosquito Control to lower their reporting threshold to us during the RNC period. We provided updates to all our partners with respect to diseases and outbreaks of public health importance occurring in our county.

\section{Results}

No epidemiologic events linked to the RNC were detected through the HCHD's enhanced surveillance that was conducted. Decreased patient volumes were seen during the RNC at our EDs closest to the convention site. No significant increases in ED visits from outside of our 5-county area were noted during the RNC. Urgent care centers reported seeing patients associated with the RNC for a variety of reasons including respiratory and gastrointestinal illness. DMAT surveillance showed mainly routine visits but four secret service agents did seek care for respiratory illness during the convention.

\section{Conclusions}

Substantial time and resources were devoted to disease surveillance in the 6 months leading up to the RNC and during the event. While no epidemiologic events were detected, the public health surveillance infrastructure has clearly been strengthened in our county. We are receiving our ED syndromic data, from many of our hospitals, every two hours as opposed to every day. We have established relationships with our urgent case centers and hope to begin receiving urgent care center data on a daily basis in the near future. Receiving DMAT data through ESSENCE could prove very useful in the future, especially in Florida where hurricanes are always a threat. Lastly the improved relationships with our health care providers should be beneficial as we move forward.

\section{Keywords}

mass gathering; national special security event; convention

\section{Acknowledgments}

Janet Hamilton, Aaron Kite-Powell, and Aaron Chern, Florida Department of Health, Bureau of Epidemiology

Cynthia Lewis-Younger and Joann Chambers-Emerson, Florida Poison Information Center -Tampa

Dina Passman, Health and Human Services

\section{References}

Hick J, Frascone R, Grimm K, Hillman M, Griffith J, Hogan M, TrotskySirr R, Branu. Health and Medical Preparedness and Response to the 2008 Republican National Convention. Disaster Medicine and Public Health Preparedness 3(4);224-232.

Kade K, Brinsfield K, Serino R, Savoia E, Koh H. Emergency Medical Consequence Planning and Management for National Special Security Events After September 11: Boston 2004., Disaster Medicine and Public Health Preparedness 2(3);166-173.

\section{*David Atrubin}

E-mail: David_Atrubin@doh.state.fl.us 